\title{
S-1
}

\section{The time course of molecular and metabolic events in the development of insulin resistance in fructose-fed rats}

\author{
Sergio Polakof ${ }^{1,2 *}$, Dominique Dardevet ${ }^{1,2}$, Bernard Lyan ${ }^{1,2,3}$, Laurent Mosoni ${ }^{1,2}$, Eva \\ Gatineau $^{1,2}$, Jean-François Martin ${ }^{1,2,3}$, Estelle Pujos-Guillot ${ }^{1,2,3}$, Andrzej Mazur ${ }^{1,2}$, Blandine \\ Comte $^{1,2}$
}

(1) Clermont Université, Université d'Auvergne, Unité de Nutrition Humaine, BP 10448,

$$
\text { F-63000 CLERMONT-FERRAND }
$$

(2) INRA, UMR 1019, UNH, CRNH Auvergne, F-63000 CLERMONT-FERRAND

(3) INRA, UMR 1019, Plateforme d'Exploration du Métabolisme, UNH, F-63000

Clermont-Ferrand, France

Table of contents

Supporting Table S-1- Gene list of genes analysed by PCR (TaqMan® Low Density Array System) 


\section{Table-S-1}

Gene list of genes analysed by PCR (TaqMan ${ }^{\circledR}$ Low Density Array System)

\begin{tabular}{|c|c|}
\hline Acaa2 & acetyl-Coenzyme A acyltransferase 2 \\
\hline Acaca & acetyl-coenzyme A carboxylase alpha \\
\hline Acly & ATP citrate lyase \\
\hline Acox 1 & acyl-Coenzyme A oxidase 1 \\
\hline Akt1 & v-akt murine thymoma viral oncogene homolog 1 \\
\hline Akt2 & v-akt murine thymoma viral oncogene homolog 2 \\
\hline Akt3 & v-akt murine thymoma viral oncogene homolog 3 (protein kinase $\mathrm{B}$, gamma) \\
\hline Apoe & apolipoprotein E \\
\hline Cat & catalase \\
\hline Cebpa & CCAAT/enhancer binding protein (C/EBP), alpha \\
\hline Cebpb & CCAAT/enhancer binding protein (C/EBP), beta \\
\hline Cptla & carnitine palmitoyltransferase 1a, liver \\
\hline Cpt2 & carnitine palmitoyltransferase 2 \\
\hline Creb1 & cAMP responsive element binding protein 1 \\
\hline Crebbp & CREB binding protein \\
\hline Cs & citrate synthase \\
\hline Eif2b1 & eukaryotic translation initiation factor $2 \mathrm{~B}$, subunit 1 alpha \\
\hline Eif4ebp1 & eukaryotic translation initiation factor $4 \mathrm{E}$ binding protein 1 \\
\hline Fabp1 & fatty acid binding protein 1 , liver \\
\hline Fabp3 & fatty acid binding protein 3 \\
\hline Fasn & fatty acid synthase \\
\hline Fbp1 & fructose-1,6-bisphosphatase 1 \\
\hline Fos & FBJ osteosarcoma oncogene \\
\hline Foxo1 & forkhead box O1, Gene rCG50041 Celera Annotation \\
\hline Foxo3 & forkhead box O3, Gene rCG58542 Celera Annotation \\
\hline Foxo4 & forkhead box $\mathrm{O} 4$ \\
\hline G6pc & glucose-6-phosphatase, catalytic subunit \\
\hline Gck & glucokinase \\
\hline Glp1r & glucagon-like peptide 1 receptor \\
\hline Gpd1 & glycerol-3-phosphate dehydrogenase 1 (soluble) \\
\hline Gpx1 & glutathione peroxidase 1 \\
\hline Gsk3b & glycogen synthase kinase 3 beta \\
\hline Gsr & glutathione reductase \\
\hline Gstk1 & glutathione S-transferase kappa 1 \\
\hline Gys2 & glycogen synthase 2 \\
\hline $\mathrm{Hk} 2$ & hexokinase 2 \\
\hline Hnf4a & hepatocyte nuclear factor 4 , alpha \\
\hline Idh1 & isocitrate dehydrogenase 1 (NADP+) \\
\hline $\operatorname{Idh} 2$ & isocitrate dehydrogenase $2(\mathrm{NADP}+)$, mitochondrial \\
\hline Igf1r & insulin-like growth factor 1 receptor,Gene rCG24751 Celera Annotation \\
\hline Igf2 & insulin-like growth factor 2 \\
\hline Igfbp1 & insulin-like growth factor binding protein 1 \\
\hline Insr & insulin receptor \\
\hline
\end{tabular}




\begin{tabular}{|c|c|}
\hline Irs1 & insulin receptor substrate 1 \\
\hline Irs2 & insulin receptor substrate 2 \\
\hline Jun & Jun oncogene \\
\hline Ldlr & low density lipoprotein receptor \\
\hline Lpl & lipoprotein lipase \\
\hline Mdh1 & malate dehydrogenase 1, NAD (soluble) \\
\hline $\operatorname{Mdh} 2$ & malate dehydrogenase 2, NAD (mitochondrial) \\
\hline Mlxipl & MLX interacting protein-like \\
\hline Mlycd & malonyl-CoA decarboxylase \\
\hline Mpo & myeloperoxidase \\
\hline Mtor & mechanistic target of rapamycin (serine/threonine kinase) \\
\hline Nfkb1 & nuclear factor of kappa light polypeptide gene enhancer in B-cells 1 \\
\hline Nos2 & nitric oxide synthase 2 , inducible \\
\hline Nr1h2 & nuclear receptor subfamily 1 , group $\mathrm{H}$, member 2, Gene rCG54656 Celera Annotation \\
\hline Nr1h3 & nuclear receptor subfamily 1 , group $\mathrm{H}$, member 3 \\
\hline Pc & pyruvate carboxylase \\
\hline Pck1 & phosphoenolpyruvate carboxykinase 1 (soluble) \\
\hline Pck2 & phosphoenolpyruvate carboxykinase 2 (mitochondrial) \\
\hline Pdha2 & pyruvate dehydrogenase (lipoamide) alpha 2 \\
\hline Pdhb & pyruvate dehydrogenase (lipoamide) beta \\
\hline Pdpk1 & 3-phosphoinositide dependent protein kinase-1 \\
\hline Pfkl & phosphofructokinase, liver \\
\hline Pik3ca & phosphoinositide-3-kinase, catalytic, alpha polypeptide \\
\hline Pik3cb & phosphoinositide-3-kinase, catalytic, beta polypeptide \\
\hline Pik3r1 & phosphoinositide-3-kinase, regulatory subunit 1 (alpha) \\
\hline Pik3r2 & phosphoinositide-3-kinase, regulatory subunit 2 (beta) \\
\hline Pklr & pyruvate kinase, liver and $\mathrm{RBC}$ \\
\hline Ppara & peroxisome proliferator activated receptor alpha \\
\hline Pparg & peroxisome proliferator-activated receptor gamma \\
\hline Ppargc1a & peroxisome proliferator-activated receptor gamma \\
\hline Ppplca & protein phosphatase 1 , catalytic subunit, alpha isoform \\
\hline Prkag1 & protein kinase, AMP-activated, gamma 1 non-catalytic subunit \\
\hline Prkag2 & protein kinase, AMP-activated, gamma 2 non-catalytic subunit \\
\hline Prkag3 & protein kinase, AMP-activated, gamma 3 non-catalytic subunit \\
\hline Prkcg & protein kinase $\mathrm{C}$, gamma \\
\hline Prkcz & protein kinase $\mathrm{C}$, zeta \\
\hline Pygl & phosphorylase, glycogen, liverGene rCG61353 Celera Annotation \\
\hline Rpl13a & ribosomal protein L13AGene rCG53885 Celera Annotation \\
\hline Rxra & retinoid $\mathrm{X}$ receptor alpha \\
\hline Rxrb & retinoid $\mathrm{X}$ receptor beta \\
\hline Rxrg & retinoid $\mathrm{X}$ receptor gamma \\
\hline Slc27a4 & solute carrier family 27 (fatty acid transporter), member 4 \\
\hline Slc2a1 & solute carrier family 2 (facilitated glucose transporter), member 1 \\
\hline Slc2a4 & solute carrier family 2 (facilitated glucose transporter), member 4 \\
\hline Sod1 & superoxide dismutase 1 , soluble \\
\hline Srebf1 & sterol regulatory element binding transcription factor 1 \\
\hline
\end{tabular}


Ucp3

Ugp2

Actb

Gapdh

$18 \mathrm{~s}$ uncoupling protein 3 (mitochondrial, proton carrier)

UDP-glucose pyrophosphorylase 2

actin, beta

glyceraldehyde-3-phosphate dehydrogenase

Ribosomal Protein S18 